University of Wollongong

Research Online

Faculty of Engineering and Information

Faculty of Engineering and Information

Sciences - Papers: Part B

Sciences

2019

A case study on pricing foreign exchange options using the modified CraigSneyd ADI scheme

Chittaranjan Mishra

Indian Institute of Technology, Ropar

Xiaoping Lu

University of Wollongong, xplu@uow.edu.au

Follow this and additional works at: https://ro.uow.edu.au/eispapers1

Part of the Engineering Commons, and the Science and Technology Studies Commons

Research Online is the open access institutional repository for the University of Wollongong. For further information contact the UOW Library: research-pubs@uow.edu.au 


\title{
A case study on pricing foreign exchange options using the modified Craig-Sneyd ADI scheme
}

\author{
Abstract \\ One of the prominent alternating direction implicit (ADI) schemes for numerically pricing financial options, \\ the modified Craig-Sneyd scheme, is put to test for its reliability and efficiency for solving non-trivial \\ problems with empirical market data. The Heston equation for pricing foreign exchange options of \\ European style, a two-dimensional convection-diffusion-reaction equation with a mixed derivative term, is \\ numerically solved for various parameter values observed in the market by employing the said scheme. \\ Numerical stability and convergence issues of this scheme is compared with another popular \\ Hundsdorfer-Verwer ADI scheme. From among a total of 56 options on 8 currency pairs, it is observed \\ that some interesting ones for which the so-called Feller condition is strongly violated, create \\ Snadditional computational challenges. Suggestions on successful implementation of the MCS scheme \\ are made in order to tackle these challenging test cases.

\section{Disciplines} \\ Engineering | Science and Technology Studies

\section{Publication Details} \\ Mishra, C. \& Lu, X. (2019). A case study on pricing foreign exchange options using the modified Craig- \\ Sneyd ADI scheme. International Journal of Computer Mathematics, Online First 1-19.
}




\title{
A case study on pricing foreign exchange options using the modified Craig-Sneyd ADI scheme
}

\author{
Chittaranjan Mishra ${ }^{\mathrm{a}}$ and Xiaoping $\mathrm{Lu}^{\mathrm{b}}$ \\ a Department of Mathematics, Indian Institute of Technology Ropar, Rupnagar, Punjab, \\ India - 140001; ${ }^{\mathrm{b}}$ School of Mathematics and Applied Statistics, University of Wollongong, \\ Wollongong, NSW 2522, Australia
}

\section{ARTICLE HISTORY}

Compiled May 1, 2019

\begin{abstract}
One of the prominent alternating direction implicit(ADI) schemes for numerically pricing financial options, the modified Craig-Sneyd scheme, is put to test for its reliability and efficiency for solving non-trivial problems with empirical market data. The Heston equation for pricing foreign exchange options of European style, a twodimensional convection-diffusion-reaction equation with a mixed derivative term, is numerically solved for various parameter values observed in the market by employing the said scheme. Numerical stability and convergence issues of this scheme is compared with another popular Hundsdorfer-Verwer ADI scheme. From among a total of 56 options on 8 currency pairs it is observed that some interesting ones for which the so-called Feller condition is strongly violated, create additional computational challenges. Suggestions on successful implementation of the MCS scheme are made in order to tackle these challenging test cases.
\end{abstract}

\section{KEYWORDS}

Foreign exchange options, convection-diffusion equations, Initial-boundary value problems, ADI schemes, stability.

\section{Introduction}

The pricing equations of contemporary financial options often involve time-dependent multidimensional partial differential equations (PDEs) containing mixed derivative terms. As analytic solutions to these PDEs are rarely available in a closed form, one looks for their approximate numerical solutions. ADI schemes are a type of timestepping numerical techniques that have recently become highly popular among finance practitioners for solving these PDEs, cf. [1, 3, 21]. There have been various ADI schemes proposed in the literature that are specifically designed for financial applications cf.[17, 23, 31]. From among a pool of ADI techniques, it is worth mentioning that, the so-called Douglas scheme [6] and the Craig-Sneyd scheme [4] are widely used by the quant community. The Modified Craig-Sneyd (MCS) scheme is yet another promising but relatively new ADI scheme that was initially proposed in [23]. It may be remarked that the Craig-Sneyd scheme[4], originally called the iterated scheme in loc. cit., is of order two only for a specific parameter value $\theta=1 / 2$ when applied to 
diffusion problems with mixed derivatives and has order one otherwise. On the other hand the MCS scheme is able to increase the order to two for any $\theta$ while retaining the favourable property of unconditional stability. Later, the MCS scheme has been applied successfully to solve financial PDEs, see [10-12, 18, 24]. While it is interesting to develop more efficient numerical techniques, it is also equally important to study their (unconditional) stability properties and convergence behaviours. In general, investigating stability of ADI schemes involves analyzing complex inequalities and deriving stability bounds that often requires non-trivial computations. The MCS scheme is analyzed for its stability, for instance in $[19,20]$, with its application to multidimensional convection-diffusion equations and many interesting results have been obtained. It is worth mentioning here that the modern option pricing models often consider underlying stochastic processes to be correlated. As it turns out, these correlations are directly reflected in the mixed derivatives of the corresponding pricing PDE. Recently, in [26], another intriguing stability result of the MCS scheme was obtained where the size of the mixed derivative coefficient is effectively taken into account.

Notably, all the above stability results are derived in the von Neumann framework where various assumptions, for instance coefficients of the PDE that need to be solved are constants, boundary conditions are periodic, discretization grids are uniform Cartesian ones and errors are measured in the $\ell^{2}$-norm, are made. Event hough all of these assumptions are vital for theoretical stability analyses, in real world scenarios one rarely sticks to these assumptions. The main aim of this paper is to study numerical implementation of the theoretical stability results of the MCS scheme to solve challenging real world problems. Although a lot of research has been done on application of various numerical techniques to the Heston PDE, most of the test cases in those studies are usually taken from existing peer literature, mainly for the ease of comparison of numerical results. In this study two very important aspects of the MCS scheme have been validated using real-life data - that the scheme is stable and robust and that the theoretical stability bounds are very sharp, thus should be strictly followed.

In this study, we price European type foreign exchange (FX) options under the Heston stochastic volatility model [14] using the MCS scheme. Heston parameters corresponding to options on eight currency pairs, as observed in the FX market, are used in the analysis. It is observed that in the FX market, the parameters in the Heston equation frequently violate the so-called Feller condition. This issue creates challenges for many well-known numerical methods for solving the Heston equation efficiently. It is an important factor why we consider FX options as our test cases for validation of the MCS scheme in this study. Furthermore, to make the test cases non-trivial, two special instruments are considered - the strangle and butterfly options, purely in view of their (relatively) higher non-smooth payoff functions. Although these options are special linear combinations of European puts and calls, they are more challenging than simple vanilla calls or puts as far as the finite difference approximation is concerned, due to the presence of extra kinks in the pay-off functions. The Hundsdorfer-Verwer (HV) scheme [5, 18] is used for numerical convergence analysis of the MCS scheme.

The remaining sections are organised as follows: in section 2, the type of FX options considered for this research are introduced. The Heston stochastic volatility model and the Heston PDE for FX options are presented in section 3. The section 4 presents spatial discretization of the Heston PDE on two different non-uniform grids, followed by time integration using two ADI schemes. All numerical experiments are conducted in section 5 that includes crude options market data and presents spatial discretization errors and the time-discretization errors. Numerous sample figures representing nu- 
merical stability and convergence issues of the two ADI schemes are also presented here. The concluding section 6 summarises important observations with a few remarks.

\section{FX options}

An FX option is a financial derivative instrument that gives its holder right, not obligation, to exchange money denominated in one currency into another currency, at a pre-agreed exchange rate on a specific date in the future. If the exchange could only take place on the date it is a European type option, and if the exchange could also be done before the date it is of American type. Unlike equity options, FX options are based on spot exchange rates which are not tradable assets. The tradable asset is the foreign bond valued in the domestic currency. As a result, there is an extra term containing the foreign interest rate in the Black-Scholes PDE for the fair values of FX options (in the risk-neutral frame-work), compared with that for equity options. A detailed description can be found in any standard book on FX options, for example $[3,25]$.

In this paper two frequently traded options are considered, one is popular when the market outlook is highly volatile, and the other is more attractive to traders in a market with very little volatility. The main aim however remains to discuss the numerical implementation of the MCS scheme for pricing FX options and compare the results with the HV scheme.

\section{Butterfly option}

A butterfly option is an option portfolio that combines four vanilla call options with the same underlying and the same expiry date but three different strike prices and is such that a buyer (holder) has a large probability of making limited profit when the market has low volatility. A long butterfly position consists of one long call with strike price $K_{1}$, one long call with strike price $K_{2}$ and two short calls with strike $K=\left(K_{1}+K_{2}\right) / 2$ with $K_{2}>K_{1}$. Often in practice $K$ is chosen close to the spot rate $S_{t}$ at time $t=0$. The pay-off from a long butterfly option, upon expiry, is

$$
\max \left(S_{T}-K_{1}, 0\right)-2 \max \left(S_{T}-K, 0\right)+\max \left(S_{T}-K_{2}, 0\right) .
$$

This strategy is adopted when the speculator and/or hedger believes that the future price of the underlying will not move far away from the spot price. The return is maximum when the price of the underlying asset remains around the middle strike price, $K$.

\section{Strangle}

A strangle is such that the holder has a large probability of making large profit when the market has high volatility. It is a combination of a put option with strike price $K_{1}$ and a call option with strike price $K_{2}\left(K_{2}>K_{1}\right)$, both on the same underlying and having the same maturity date. On maturity, the payoff from a long strangle is

$$
\max \left(K_{1}-S_{T}, 0\right)+\max \left(S_{T}-K_{2}, 0\right) .
$$


In contrast to the butterfly option strategy, a strangle option position is adopted if the investor believes that there will be large movement in the future price of the underlying asset (in our case the FX rate), but not sure in which direction.

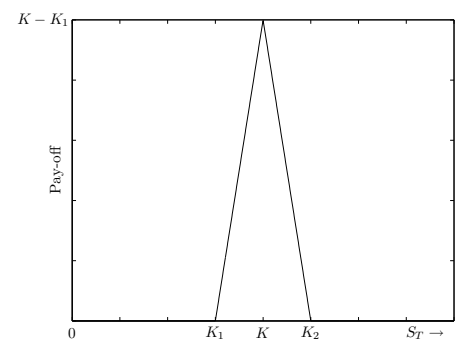

(a) butterfly

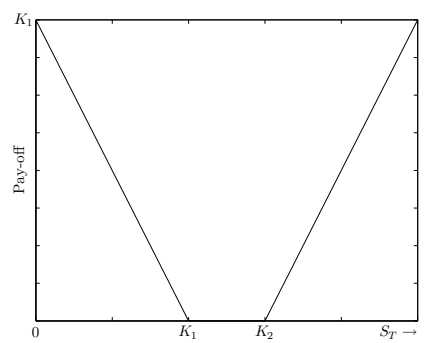

(b) strangle

Figure 1. Non-smooth pay-offs from long positions.

\section{Stochastic volatility and the Heston equation}

\subsection{The Heston stochastic volatility model}

The following asset price model was proposed by Heston, cf.[14], as an extension to the Black-Scholes model. Assume that the spot rate process $S_{t}(0 \leq t \leq T)$ is given by the stochastic differential equation

$$
d S_{t}=\mu S_{t} d t+\sqrt{V_{t}} S_{t} d W_{t}^{(1)}
$$

where the instantaneous variance $V_{t}$ is given by

$$
d V_{t}=\kappa\left(\eta-V_{t}\right) d t+\sigma \sqrt{V_{t}} d W_{t}^{(2)}
$$

Here $W_{t}^{(1)}, W_{t}^{(2)}$ are two Wiener processes with correlation $\rho \in(-1,1)$. The parameters $\mu, \sigma, \kappa$ and $\eta$ represent the asset rate of return, vol-of-variance, mean reversion rate and mean reversion level, respectively.

\section{The Feller condition}

The variance process (2) involves a square root term. It is necessary to have the argument of the square root nonnegative. The Feller condition is said to be satisfied if $2 \kappa \eta>\sigma^{2}$. Fulfilment of the Feller condition is sufficient for $V_{t}$ to remain strictly positive:

Lemma 3.1. Let $V_{0}>0$ and $2 \kappa \eta>\sigma^{2}$. Then $V_{t}>0, \forall t>0$

(cf. the original work [8] and for more discussions, e.g., [3, 25]). However, the Feller condition is only rarely satisfied in the market, which can be observed clearly from the negative values of $q$ (defined in (20)) in Table 1. If the Feller condition is violated, then $V_{t}$ could become zero. This will ultimately lead to the option pricing equation becoming convection-reaction equation, which may pose problems for numerical solutions by standard methods. 


\subsection{The Heston PDE}

Let the spot FX rate be $s$ and its variance be $v$ at time $T-t$ ( $t$ being the time to expiry). Denote the fair price of a European option by $u(s, v, t)$. Then, by applying the risk-neutral valuation technique and assuming that the market price of volatility risk equals zero, it can be shown that, cf.[14], $u$ satisfies

$$
\frac{\partial u}{\partial t}=\frac{1}{2} s^{2} v \frac{\partial^{2} u}{\partial s^{2}}+\rho \sigma s v \frac{\partial^{2} u}{\partial s \partial v}+\frac{1}{2} \sigma^{2} v \frac{\partial^{2} u}{\partial v^{2}}+\left(r_{d}-r_{f}\right) s \frac{\partial u}{\partial s}+\kappa(\eta-v) \frac{\partial u}{\partial v}-r_{d} u
$$

for $0<t \leq T, s>0, v>0$, where $r_{d}$ and $r_{f}$ represent the risk-neutral domestic and foreign interest rates, respectively.

The Heston PDE (3) is a time-dependent two-dimensional convection-diffusionreaction equation with a mixed derivative on the unbounded spatial domain $(0, \infty) \times$ $(0, \infty)$. In general, when solving such initial boundary value problems we must specify boundary conditions as well as an initial condition. We will discuss the conditions separately as follows.

\section{Initial conditions}

The payoff function of a European option determines the initial condition for the PDE (3). Thus the initial condition for the European butterfly option is

$$
u(s, v, 0)=\max \left(s-K_{1}, 0\right)-2 \max (s-K, 0)+\max \left(s-K_{2}, 0\right)
$$

and that for the European strangle is

$$
u(s, v, 0)=\max \left(K_{1}-s, 0\right)+\max \left(s-K_{2}, 0\right) .
$$

\section{Boundary conditions}

In order to solve the PDE (3) numerically, we need to truncate the unbounded domain to a bounded set $\left[0, S_{\max }\right] \times\left[0, V_{\max }\right]$, where $S_{\max }$ and $V_{\max }$ should be sufficiently large to avoid any boundary effect. In this paper, $S_{\max }$ and $V_{\max }$ are chosen empirically as listed in Table 3. In general, we must define boundary conditions at all boundaries. At $s=0, s=S_{\max }$ and $v=V_{\max }$, the following boundary conditions are applied:

For the European butterfly option: $\left\{\begin{array}{l}u(0, v, t)=0, \\ \frac{\partial u}{\partial s}\left(S_{\max }, v, t\right)=0, \\ u\left(s, V_{\max }, t\right)=0 .\end{array}\right.$

For the European strangle option: $\left\{\begin{array}{l}u(0, v, t)=K_{1} e^{-r_{d} t}, \\ \frac{\partial u}{\partial s}\left(S_{\max }, v, t\right)=e^{-r_{f} t}, \\ u\left(s, V_{\max }, t\right)=K_{1} e^{-r_{d} t}+s e^{-r_{f} t} .\end{array}\right.$ 
The boundary condition along $v=0$ is an issue that needs to be discussed in more detail. Mathematically, the Fichera function [9] for the Heston PDE (3) can be shown to equal $\kappa \eta-\frac{1}{2} \sigma^{2}$ on $v=0$. Here it is worth noting the equivalence between the Feller condition $\left(\kappa \eta-\frac{1}{2} \sigma^{2}>0\right)$ and the Fichera function at $v=0$, which is also mentioned in $[2,31]$ and many others. According to the Fichera theory, if $\kappa \eta \geq \frac{1}{2} \sigma^{2}$ (or $q \geq 0$ ), no boundary condition should be prescribed; if $\kappa \eta<\frac{1}{2} \sigma^{2}$ (or $q<0$ ) a boundary condition is needed, however, the theory does not specify what condition is to be prescribed. As a result, there is no unified approach for the boundary condition at $v=0$. In general, a combination of mathematical, financial and heuristic reasoning allows us to find consistent and acceptable boundary condition for a problem as pointed out in [7].

On the other hand, a boundary condition is always required at $v=0$ for any numerical approximation method. A typical approach is to simply let the Heston PDE (3) be satisfied along $v=0[18,30]$. Another good reference is [31], where a boundary condition (the payoff function) is prescribed, based on financial implications, for American options regardless of the fulfillment of the Feller condition.

In our test cases, there are some positive values and some negative values of $q$ as shown in Table 1. Theoretically, a boundary condition should be prescribed for the cases with $q<0$, and none for the other cases. Here we choose not to have any 'explicit' boundary condition, instead following the approach in $[18,30]$. At $v=0$, the Heston PDE (3) degenerates to a hyperbolic PDE:

$$
\frac{\partial u}{\partial t}=\left(r_{d}-r_{f}\right) s \frac{\partial u}{\partial s}+\kappa \eta \frac{\partial u}{\partial v}-r_{d} u
$$

The equation (8) is allowed to hold along $v=0$ as the boundary condition for our numerical solution. It is worth noting that requiring the pricing PDE to be satisfied at $v=0$ is also the practice in the original Heston paper [14]. It is also worthwhile to mention that to 'validate' our choice of boundary condition we tested the effect of taking an 'explicit' boundary condition at $v=0$, say, $\frac{\partial u}{\partial v}=0$, for cases where the Feller condition is either satisfied or violated. Our experimental results show that there is hardly any difference between the results for cases with an 'explicit' boundary condition and those using (8) as the boundary condition, which also proves the strength of the MCS ADI scheme.

However, extra care needs to be taken when the above boundary condition is applied, as using the central finite difference approximation at this boundary where extra ghost-points are needed to the left of $v=0$ could lead to oscillations, cf. [16, 27]. This oscillatory behaviour is more visible in our trials for the cases where the Feller condition is strongly violated $(q<0)$. Therefore, in our numerical solutions we adopt one-sided divided difference at this boundary, the values are computed implicitly without noticeable oscillations. Numerical implementation of all boundary conditions is discussed further in the next section.

\section{Numerical solution of the Heston PDE}

In order to obtain numerical solutions of the initial boundary value problems (3), (4), (6) and (3), (5), (7), the Heston PDE (3) is first semi-discretized on a Cartesian grid by the finite difference scheme in [18]. 


\subsection{Spatial discretization}

Non-uniform grid: s-dimension

The initial functions (4) and (5) are non-smooth as they have discontinuous first derivatives at $s=K_{1}, K, K_{2}$ and $s=K_{1}, K_{2}$, respectively. In order to increase the accuracy of the numerical solutions, more mesh points are needed near those non-smooth points. From a practical point of view, a region $\left[S_{\text {left }}, S_{\text {right }}\right] \subset\left[0, S_{\max }\right]$ containing $K_{1}, K, K_{2}$ is where one wishes to obtain option prices. Thus, meshes of different densities in the $s$-dimension, dense and uniform inside, sparse and non-uniform outside of the interval $\left[S_{\text {left }}, S_{\text {right }}\right]$, are adapted to make the computation more efficient.

For the butterfly option, a mesh is generated by adapting the technique in [10] as follows. Consider equidistant points $\xi_{\min }=\xi_{0}<\xi_{1}<\ldots<\xi_{m_{1}}=\xi_{\max }$ defined by

$$
\begin{aligned}
\xi_{\text {min }} & =\sinh ^{-1}\left(\frac{-S_{\text {left }}}{d_{1}}\right), \\
\xi_{\text {int }} & =\frac{S_{\text {right }}-S_{\text {left }}}{d_{1}}, \\
\text { and } \quad \xi_{\text {max }} & =\xi_{\text {int }}+\sinh ^{-1}\left(\frac{S_{\text {max }}-S_{\text {right }}}{d_{1}}\right),
\end{aligned}
$$

where $m_{1} \geq 1$ is a given integer and $d_{1}>0$ is a free parameter. Then the mesh $0=s_{0}<s_{1}<\ldots<s_{m_{1}}=S_{\max }$ is given by

$$
s_{i}= \begin{cases}S_{\text {left }}+d_{1} \sinh \left(\xi_{i}\right), & \xi_{\text {min }} \leq \xi_{i}<0 \\ S_{\text {left }}+d_{1} \xi_{i}, & 0 \leq \xi_{i} \leq \xi_{\text {int }} \\ S_{\text {right }}+d_{1} \sinh \left(\xi_{i}-\xi_{\text {int }}\right), & \xi_{\text {int }}<\xi_{i} \leq \xi_{\text {max }}\end{cases}
$$

The non-uniform mesh for the the strangle option is generated by following the technique in $[18,29]$. Here the mesh points are more dense around $s=K$, the middle of the two strikes $K_{1}, K_{2}$. Let $m_{1}, d_{1}$ be as above and equidistant points $\xi_{\min }=\xi_{0}<$ $\xi_{1}<\ldots<\xi_{m_{1}}=\xi_{\max }$ be given by

$$
\xi_{i}=\sinh ^{-1}\left(\frac{-K}{d_{1}}\right)+i \Delta \xi \quad\left(0 \leq i \leq m_{1}\right)
$$

where

$$
\Delta \xi=\frac{1}{m_{1}}\left[\sinh ^{-1}\left(\left(S_{\max }-K\right) / d_{1}\right)-\sinh ^{-1}\left(-K / d_{1}\right)\right] .
$$

Then the non-uniform mesh $0=s_{0}<s_{1}<\ldots<s_{m_{1}}=S_{\max }$ is defined by

$$
s_{i}=K+d_{1} \sinh \left(\xi_{i}\right) \quad\left(0 \leq i \leq m_{1}\right) .
$$

As can be seen from (9) and (10) the parameter $d_{1}$ controls the density of mesh points in the region of interest. Let us define $\Delta \xi=\xi_{i}-\xi_{i-1}$ and $\Delta s_{i}=s_{i}-s_{i-1}$ for 


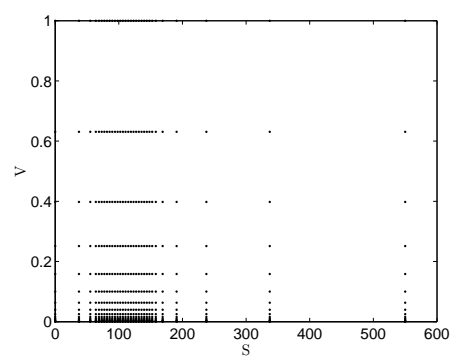

(a) butterfly option

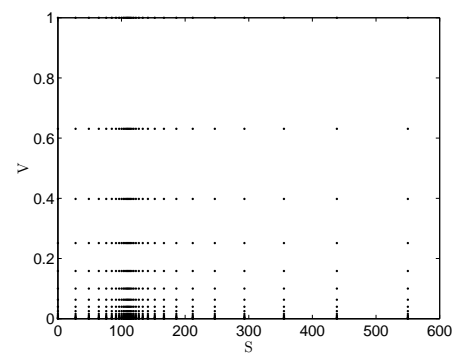

(b) strangle option

Figure 2. Sample grids

any $0<i \leq m_{1}$. If there exist real constants $C_{0}, C_{1}, C_{2}>0$ such that

$$
C_{0} \Delta \xi \leq \Delta s_{i} \leq C_{1} \Delta \xi \quad \text { and } \quad\left|\Delta s_{i+1}-\Delta s_{i}\right| \leq C_{2}(\Delta \xi)^{2}
$$

hold uniformly in $i, m_{1}$, we say that the mesh is smooth. It can be verified that both the meshes defined above are smooth.

\section{Non-uniform grid: v-dimension}

A relatively dense mesh is chosen at $v \approx 0$ for the following reasons. Firstly the region $v \approx 0$ is of practical interest, as the volatility of the spot rate process lies mostly in this region. Secondly, the Heston PDE is convection dominated in the $v$-direction for $v \approx 0$. In addition, the initial function is non-smooth. All these factors combined could yield numerical inaccuracies at $v \approx 0$.

We choose a non-uniform mesh in the $v$-dimension analogous to that of the $s$ dimension for the strangle option. Let $m_{2} \geq 1$ be any given integer and $d_{2}>0$ be a given constant. Let the equidistant points $\zeta_{j}=j \cdot \Delta \zeta\left(j=0,1, \ldots, m_{2}\right)$ be defined by

$$
\Delta \zeta=\frac{1}{m_{2}} \sinh ^{-1}\left(V_{\max } / d_{2}\right) .
$$

Define the mesh $0=v_{0}<v_{1}<\cdots<v_{m_{2}}=V_{\max }$ by the transformation

$$
v_{j}=d_{2} \sinh \left(\zeta_{j}\right), \quad j=0,1, \ldots, m_{2} .
$$

This mesh is also smooth. The control parameter $d_{2}$ determines the density of mesh points near $v=0$.

In our experiment we choose the control parameters as $d_{1}=K / 100$ in $(9), d_{1}=$ $K / 10$ in (10) and $d_{2}=V_{\max } / 500$ in (12). Figures (2a), (2b) display sample grids generated for the two options for $m_{1}=2 m_{2}=30, K=110, S_{\max }=5 K, V_{\max }=$ $1, S_{\text {left }}=70, S_{\text {right }}=150$. Both figures demonstrate the relatively large number of grid points in the region of interest.

\section{Cell averaging}

It is possible that the critical points $K_{1}, K, K_{2}$ do not lie exactly on the $s$-mesh defined above. This introduces a so-called quantization error. Cell averaging is a well-known technique to reduce the quantization error, which uses the perception that the value 
of a function on a grid point represents the average value of the function over a surrounding cell, cf. [29, Chapter 4]. Thus, we use cell averaged values at the mesh points in the $s$-dimension that are nearest to the critical points, and sampled values everywhere on the grid.

In case of a call option, for example, if $s_{i}$ is the nearest mesh point to its strike $K$, then we replace the value of the initial function $u$ at $s_{i}$ by the cell average

$$
\frac{1}{h} \int_{s_{i-1 / 2}}^{s_{i+1 / 2}} \max (s-K, 0) d s,
$$

where $s_{i-1 / 2}=\frac{1}{2}\left(s_{i-1}+s_{i}\right), s_{i+1 / 2}=\frac{1}{2}\left(s_{i}+s_{i+1}\right)$ and $h=s_{i+1 / 2}-s_{i-1 / 2}$.

\section{FD discretization}

We approximate the spatial derivatives in the Heston PDE (3) using finite difference discretization as follows. Let $\psi: \mathbb{R} \rightarrow \mathbb{R}$ be any given sufficiently often continuously differentiable function. Let a mesh be given by $x_{0}<x_{1}<\cdots<x_{m}$ with $\Delta x_{i}=$ $x_{i}-x_{i-1}$. We define

$$
\begin{aligned}
& \psi^{\prime}\left(x_{i}\right) \approx \alpha_{i,-2} \psi\left(x_{i-2}\right)+\alpha_{i,-1} \psi\left(x_{i-1}\right)+\alpha_{i, 0} \psi\left(x_{i}\right), \\
& \psi^{\prime}\left(x_{i}\right) \approx \beta_{i,-1} \psi\left(x_{i-1}\right)+\beta_{i, 0} \psi\left(x_{i}\right)+\beta_{i, 1} \psi\left(x_{i+1}\right), \\
& \psi^{\prime}\left(x_{i}\right) \approx \gamma_{i, 0} \psi\left(x_{i}\right)+\gamma_{i, 1} \psi\left(x_{i+1}\right)+\gamma_{i, 2} \psi\left(x_{i+2}\right),
\end{aligned}
$$

where the coefficients are given by

$$
\begin{aligned}
& \alpha_{i,-2}=\frac{\Delta x_{i}}{\Delta x_{i-1}\left(\Delta x_{i-1}+\Delta x_{i}\right)}, \quad \alpha_{i,-1}=\frac{-\Delta x_{i-1}-\Delta x_{i}}{\Delta x_{i-1} \Delta x_{i}}, \quad \alpha_{i, 0}=\frac{\Delta x_{i-1}+2 \Delta x_{i}}{\Delta x_{i}\left(\Delta x_{i-1}+\Delta x_{i}\right)}, \\
& \beta_{i,-1}=\frac{-\Delta x_{i+1}}{\Delta x_{i}\left(\Delta x_{i}+\Delta x_{i+1}\right)}, \quad \beta_{i, 0}=\frac{\Delta x_{i+1}-\Delta x_{i}}{\Delta x_{i} \Delta x_{i+1}}, \quad \beta_{i, 1}=\frac{\Delta x_{i}}{\Delta x_{i+1}\left(\Delta x_{i}+\Delta x_{i+1}\right)}, \\
& \gamma_{i, 0}=\frac{-2 \Delta x_{i+1}-\Delta x_{i+2}}{\Delta x_{i+1}\left(\Delta x_{i+1}+\Delta x_{i+2}\right)}, \quad \gamma_{i, 1}=\frac{\Delta x_{i+1}+\Delta x_{i+2}}{\Delta x_{i+1} \Delta x_{i+2}}, \quad \gamma_{i, 2}=\frac{-\Delta x_{i+1}}{\Delta x_{i+2}\left(\Delta x_{i+1}+\Delta x_{i+2}\right)} \text {. }
\end{aligned}
$$

To approximate the second derivative $\psi^{\prime \prime}\left(x_{i}\right)$, we use the central FD scheme

$$
\psi^{\prime \prime}\left(x_{i}\right) \approx \delta_{i,-1} \psi\left(x_{i-1}\right)+\delta_{i, 0} \psi\left(x_{i}\right)+\delta_{i, 1} \psi\left(x_{i+1}\right)
$$

where

$$
\delta_{i,-1}=\frac{2}{\Delta x_{i}\left(\Delta x_{i}+\Delta x_{i+1}\right)}, \quad \delta_{i, 0}=\frac{-2}{\Delta x_{i} \Delta x_{i+1}}, \quad \delta_{i, 1}=\frac{2}{\Delta x_{i+1}\left(\Delta x_{i}+\Delta x_{i+1}\right)} .
$$

Next we approximate the mixed derivative. Let $\psi: \mathbb{R}^{2} \rightarrow \mathbb{R}$ be any given function of two variables $x$ and $y$ that is sufficiently often continuously differentiable. Let $x_{i}, \Delta x_{i}$ be as above. Let the mesh points in the $y$-direction be given by $y_{0}<y_{1}<\ldots<y_{n}$ and $\Delta y_{j}=y_{j}-y_{j-1}$. Let $\hat{\beta}_{i, k}$ denote the coefficients in the $y$-direction analogous to $\beta_{i, k}$ in (13b). We discretize the mixed derivative $\psi_{x y}(x, y)$ by the central FD scheme

$$
\frac{\partial^{2} \psi}{\partial x \partial y}\left(x_{i}, y_{j}\right) \approx \sum_{k, l=-1}^{1} \beta_{i, k} \hat{\beta}_{j, l} \psi\left(x_{i+k}, y_{j+l}\right) .
$$


The approximation (15) can be viewed as successive application of (13b) in the $x$ - and $y$ - directions. Each of the central schemes (13b), (14), (15) and the upwind schemes (13a), (13c) has second order truncation error provided that the meshes in both the $x$ - and the $y$ - directions are smooth, in the sense of (11).

In view of the Dirichlet boundary conditions at $s=0$ and $v=V_{\max }$ in (6) and (7), the grid in $\left[0, S_{\max }\right] \times\left[0, V_{\max }\right]$ is

$$
G=\left\{\left(s_{i}, v_{j}\right): 1 \leq i \leq m_{1}, 0 \leq j \leq m_{2}-1\right\} .
$$

The spatial derivatives in (3) are discretized by the central FD schemes (13b), (14), (15) at this grid except the region $v>1$ and the boundaries $v=0$ and $s=S_{\max }$.

In the region $v>1$ the derivative $\partial u / \partial v$ is replaced by the upwind scheme (13a) whenever the flow in the $v$-direction is towards $v=V_{\max }$. This is done to avoid spurious oscillations in the FD solution when the vol-of-variance $\sigma$ is close to zero.

Similarly, at the boundary $v=0$, we apply the upwind scheme (13c) to approximate the partial derivative $\frac{\partial u}{\partial v}$ in (8) to avoid possible oscillation.

In (6) as well as (7), the Neumann boundary condition at $s=S_{\max }$ directly gives $\partial u / \partial s$. Further, it implies that the mixed derivative $\partial^{2} u / \partial s \partial v$ vanishes here. Next, we approximate the second derivative $\partial^{2} u / \partial s^{2}$ using the central scheme (14). In fact, we need the point $S_{\max }+\Delta s_{m_{1}}$ from outside the grid so as to apply the central scheme. The value at this fictitious point is obtained by extrapolation using the boundary condition at $s=S_{\max }$.

The FD discretization reduces each of the two initial boundary value problems (3), $(4),(6)$ and $(3),(5),(7)$ to a large system of ODEs of the form

$$
U^{\prime}(t)=A U(t)+g(t) \quad(0 \leq t \leq T), \quad U(0)=U_{0} .
$$

Here $A$ is a known $m \times m$ matrix and $g(t)(t>0), U_{0}$ are given $m$-vectors, with $m=m_{1} \times m_{2}$. The vector function $g$ is determined by the boundary condition (6) or (7), whereas $U_{0}$ is obtained from the initial condition (4) (resp. (5)). The vector $U(t) \in \mathbb{R}^{m}$ is unknown for $t>0$.

\subsection{Temporal discretization - the MCS and the HV schemes}

A crucial step to arrive at a robust and efficient numerical scheme to solve the ODE system (16) is to choose an efficient time-discretization method. ADI schemes are advantageous over classical time stepping schemes in meeting this goal, as has been discussed in various literatures cited in this paper. In this study, the MCS and the HV schemes are used for computation and comparison.

Following the idea in ADI techniques, we decompose the matrix $A$ in (16) into three parts,

$$
A=A_{0}+A_{1}+A_{2} .
$$

In the MCS and HV schemes the $A_{0}$ part, representing all mixed derivative terms, is always treated in an explicit fashion. While the $A_{j}$ parts for $j=1,2$ are successively treated in an implicit fashion, as in the original ADI schemes.

The vector $g(t)$ in (16) is split accordingly as

$$
g(t)=g_{0}(t)+g_{1}(t)+g_{2}(t)
$$


and define

$$
F_{j}(t, w)=A_{j} w+g_{j}(t) \quad \text { for } 0 \leq t \leq T, w \in \mathbb{R}^{m} .
$$

Once the value for the parameter $\theta$ in the following schemes is known, the ODE system (16) can be numerically solved by the MCS and HV schemes that generate successive approximations $U_{n}$ to $U\left(t_{n}\right), n=1,2,3 \ldots$, where $t_{n}=n \Delta t$ are the temporal grid points for $n \geq 0$ and $\Delta t>0$.

Modified Craig-Sneyd (MCS) scheme

$$
\left\{\begin{array}{l}
Y_{0}=U_{n-1}+\Delta t F\left(t_{n-1}, U_{n-1}\right), \\
Y_{j}=Y_{j-1}+\theta \Delta t\left(F_{j}\left(t_{n}, Y_{j}\right)-F_{j}\left(t_{n-1}, U_{n-1}\right)\right), \quad j=1,2, \\
\widehat{Y}_{0}=Y_{0}+\theta \Delta t\left(F_{0}\left(t_{n}, Y_{2}\right)-F_{0}\left(t_{n-1}, U_{n-1}\right)\right), \\
\widetilde{Y}_{0}=\widehat{Y}_{0}+\left(\frac{1}{2}-\theta\right) \Delta t\left(F\left(t_{n}, Y_{2}\right)-F\left(t_{n-1}, U_{n-1}\right)\right), \\
\widetilde{Y}_{j}=\widetilde{Y}_{j-1}+\theta \Delta t\left(F_{j}\left(t_{n}, \widetilde{Y}_{j}\right)-F_{j}\left(t_{n-1}, U_{n-1}\right)\right), \quad j=1,2, \\
U_{n}=\widetilde{Y}_{2}
\end{array}\right.
$$

Hundsdorfer-Verwer (HV) scheme

$$
\left\{\begin{array}{l}
Y_{0}=U_{n-1}+\Delta t F\left(t_{n-1}, U_{n-1}\right) \\
Y_{j}=Y_{j-1}+\theta \Delta t\left(F_{j}\left(t_{n}, Y_{j}\right)-F_{j}\left(t_{n-1}, U_{n-1}\right)\right), \quad j=1,2 \\
\widetilde{Y}_{0}=Y_{0}+\frac{1}{2} \Delta t\left(F\left(t_{n}, Y_{2}\right)-F\left(t_{n-1}, U_{n-1}\right)\right), \\
\widetilde{Y}_{j}=\widetilde{Y}_{j-1}+\theta \Delta t\left(F_{j}\left(t_{n}, \widetilde{Y}_{j}\right)-F_{j}\left(t_{n}, Y_{2}\right)\right), \quad j=1,2 \\
U_{n}=\widetilde{Y}_{2}
\end{array}\right.
$$

When $\theta=\frac{1}{2}$, the MCS scheme is equivalent to the original Craig-Sneyd scheme in [4]. The Craig-Sneyd scheme is of classical order two only for $\theta=\frac{1}{2}$, whereas the MCS scheme is of order two for arbitrary $\theta$.

Several unconditional stability results for the MCS scheme (18), when applied to multidimensional convection-diffusion equations, have been obtained in [19], [20], [23]. The latest and most relevant result to the present study [26] is discussed and employed in Section 5.2 below.

In view of applications of previously obtained stability bounds (e.g., in $[22,23])$ of several ADI schemes to problems in finance, one of the well known work is recently done in [18]. There the authors compared several ADI schemes for pricing European call options under the Heston model and concluded that the MCS scheme with $\theta=1 / 3$ is a particularly effective ADI scheme. However, the authors considered only those 
problems where the Feller condition is satisfied. The question that remains open is if the ADI schemes perform well when the Feller condition is strongly violated. In the next section we shall apply the MCS scheme to a collection of test cases where the Feller condition is not satisfied.

\section{Numerical experiments}

In our experiments, we consider a total of $56 \mathrm{FX}$ options corresponding to 8 currency pairs, each with 7 different maturities varying from 3 months up to 5 years. The standard quote for a currency pair CCFCCD represents the price of 1 unit of CCF (foreign currency) in CCD (domestic currency). The market observed Heston parameter sets (September 2008) for the options are listed in Table 1 [3, Chapter 6].

Define

$$
q:=\frac{2 \kappa \eta}{\sigma^{2}}-1
$$

A positive $q$ value indicates that the Feller condition is satisfied, whereas a negative $q$ means that the condition is violated. Note that for the majority of our test cases, the Feller condition is violated as shown in the last column of Table 1.

Values of $r_{d}$ and $r_{f}$ were not provided in the original reference [3]. Table 2 quotes representative values of interest rates during the period of September 2008, obtained from www.tradingeconomics.com.

\subsection{Spatial discretization error}

In this section we discuss the convergence of FD discretization (16) of the Heston PDE for the sets of market data specified in the Table 1, following [18]. Given mesh points $m_{1}, m_{2}$ in the $s$ - and $v$-directions, respectively, we define the global spatial discretization error at time $t=T$ by

$$
e\left(m_{1}, m_{2}\right)=\max \left\{\left|u\left(s_{i}, v_{j}, T\right)-U_{k}(T)\right|: \frac{K}{2}<s_{i}<\frac{3 K}{2}, 0<v_{j}<1\right\} .
$$

Here $u$ denotes the exact solution to either the initial boundary value problem (3), (4),(6) or (3),(5),(7) and $U_{k}$ denotes the component of the exact solution $U$ to the ODE system (16) corresponding to the grid point $\left(s_{i}, v_{j}\right)$. We note that for most cases of practical interest $(s, v) \in\left(\frac{1}{2} K, \frac{3}{2} K\right) \times(0,1)$. The modelling error that was introduced by restricting the domain of the Heston PDE to a bounded set is contained in $e\left(m_{1}, m_{2}\right)$. The contribution of the modelling error is negligible in our experiments if $S_{\max }$ and $V_{\max }$ are chosen sufficiently large. The non-uniform grids do not increase computation cost.

In the case of a European call option, the exact solution $u$ to the corresponding Heston PDE is given by the semi-analytic Heston formula [14]. Employing this formula and by applying put-call parity, one easily obtains semi-analytic pricing formulas for butterfly and strangle options. The single integrals occurring in this formula are calculated by using a numerical quadrature rule. ${ }^{1}$ To obtain a sufficiently accurate

\footnotetext{
${ }^{1}$ Thanks to Dr. Sven Foulon for providing us an exact Heston pricer code.
} 
Table 1. FX market data

\begin{tabular}{|c|c|c|c|c|c|c|c|}
\hline Case No. & Currency pair & Maturity & $\rho$ & $\sigma$ & $\kappa$ & $\eta$ & $q$ \\
\hline 01 & EURUSD & $3 \mathrm{M}$ & -0.13 & 0.49 & 6.02 & 0.02 & 0.0029 \\
\hline 02 & EURUSD & $6 \mathrm{M}$ & -0.13 & 0.41 & 3.02 & 0.02 & -0.2814 \\
\hline 03 & EURUSD & $1 Y$ & -0.13 & 0.31 & 1.50 & 0.02 & -0.3757 \\
\hline 04 & EURUSD & $2 \mathrm{Y}$ & -0.14 & 0.20 & 0.75 & 0.02 & -0.2500 \\
\hline 05 & EURUSD & $3 \mathrm{Y}$ & -0.15 & 0.16 & 0.50 & 0.02 & -0.2188 \\
\hline 06 & EURUSD & $4 Y$ & -0.16 & 0.14 & 0.38 & 0.01 & -0.6122 \\
\hline 07 & EURUSD & $5 \mathrm{Y}$ & -0.17 & 0.12 & 0.30 & 0.01 & -0.5833 \\
\hline 08 & USDJPY & $3 \mathrm{M}$ & -0.60 & 0.89 & 6.02 & 0.03 & -0.5440 \\
\hline 09 & USDJPY & $6 \mathrm{M}$ & -0.64 & 0.72 & 3.02 & 0.02 & -0.7670 \\
\hline 10 & USDJPY & $1 Y$ & -0.67 & 0.62 & 1.50 & 0.02 & -0.8439 \\
\hline 11 & USDJPY & $2 Y$ & -0.70 & 0.46 & 0.75 & 0.02 & -0.8582 \\
\hline 12 & USDJPY & $3 \mathrm{Y}$ & -0.71 & 0.41 & 0.50 & 0.02 & -0.8810 \\
\hline 13 & USDJPY & $4 \mathrm{Y}$ & -0.71 & 0.40 & 0.38 & 0.02 & -0.9050 \\
\hline 14 & USDJPY & $5 \mathrm{Y}$ & -0.71 & 0.39 & 0.30 & 0.02 & -0.9211 \\
\hline 15 & GBPUSD & $3 \mathrm{M}$ & -0.23 & 0.51 & 6.02 & 0.02 & -0.0742 \\
\hline 16 & GBPUSD & $6 \mathrm{M}$ & -0.22 & 0.38 & 3.02 & 0.02 & -0.1634 \\
\hline 17 & GBPUSD & $1 \mathrm{Y}$ & -0.22 & 0.29 & 1.50 & 0.01 & -0.6433 \\
\hline 18 & GBPUSD & $2 \mathrm{Y}$ & -0.22 & 0.20 & 0.75 & 0.01 & -0.6250 \\
\hline 19 & GBPUSD & $3 Y$ & -0.21 & 0.16 & 0.50 & 0.01 & -0.6094 \\
\hline 20 & GBPUSD & $4 Y$ & -0.21 & 0.14 & 0.38 & 0.01 & -0.6122 \\
\hline 21 & GBPUSD & $5 \mathrm{Y}$ & -0.20 & 0.11 & 0.30 & 0.01 & -0.5041 \\
\hline 22 & EURGBP & $3 \mathrm{M}$ & 0.23 & 0.37 & 6.02 & 0.02 & -0.1205 \\
\hline 23 & EURGBP & $6 \mathrm{M}$ & 0.22 & 0.29 & 3.02 & 0.02 & -0.2818 \\
\hline 24 & EURGBP & $1 Y$ & 0.23 & 0.21 & 1.50 & 0.01 & -0.3197 \\
\hline 25 & EURGBP & $2 \mathrm{Y}$ & 0.31 & 0.13 & 0.75 & 0.01 & -0.1124 \\
\hline 26 & EURGBP & $3 \mathrm{Y}$ & 0.27 & 0.09 & 0.50 & 0.01 & 0.2346 \\
\hline 27 & EURGBP & $4 Y$ & 0.26 & 0.07 & 0.38 & 0.01 & 0.5510 \\
\hline 28 & EURGBP & $5 \mathrm{Y}$ & 0.20 & 0.06 & 0.30 & 0.01 & 0.6667 \\
\hline 29 & AUDUSD & $3 \mathrm{M}$ & -0.39 & 0.76 & 6.02 & 0.04 & -0.1662 \\
\hline 30 & AUDUSD & $6 \mathrm{M}$ & -0.42 & 0.56 & 3.02 & 0.03 & -0.4222 \\
\hline 31 & AUDUSD & $1 \mathrm{Y}$ & -0.45 & 0.43 & 1.50 & 0.03 & -0.5133 \\
\hline 32 & AUDUSD & $2 \mathrm{Y}$ & -0.46 & 0.26 & 0.75 & 0.03 & -0.3343 \\
\hline 33 & AUDUSD & $3 \mathrm{Y}$ & -0.43 & 0.20 & 0.50 & 0.02 & -0.5000 \\
\hline 34 & AUDUSD & $4 \mathrm{Y}$ & -0.42 & 0.17 & 0.38 & 0.02 & -0.4740 \\
\hline 35 & AUDUSD & $5 \mathrm{Y}$ & -0.40 & 0.15 & 0.30 & 0.02 & -0.4667 \\
\hline 36 & USDBRL & $3 \mathrm{M}$ & 0.43 & 0.90 & 6.02 & 0.03 & -0.5541 \\
\hline 37 & USDBRL & $6 \mathrm{M}$ & 0.46 & 0.63 & 3.02 & 0.02 & -0.6956 \\
\hline 38 & USDBRL & $1 \mathrm{Y}$ & 0.45 & 0.44 & 1.50 & 0.02 & -0.6901 \\
\hline 39 & USDBRL & $2 \mathrm{Y}$ & 0.44 & 0.31 & 0.75 & 0.03 & -0.5317 \\
\hline 40 & USDBRL & $3 \mathrm{Y}$ & 0.42 & 0.26 & 0.50 & 0.03 & -0.5562 \\
\hline 41 & USDBRL & $4 Y$ & 0.42 & 0.22 & 0.38 & 0.03 & -0.5289 \\
\hline 42 & USDBRL & $5 \mathrm{Y}$ & 0.41 & 0.20 & 0.30 & 0.04 & -0.4000 \\
\hline 43 & AUDJPY & $3 \mathrm{M}$ & -0.50 & 1.13 & 6.02 & 0.05 & -0.5285 \\
\hline 44 & AUDJPY & $6 \mathrm{M}$ & -0.55 & 0.96 & 3.02 & 0.05 & -0.6723 \\
\hline 45 & AUDJPY & $1 \mathrm{Y}$ & -0.58 & 0.97 & 1.50 & 0.05 & -0.8406 \\
\hline 46 & AUDJPY & $2 \mathrm{Y}$ & -0.55 & 0.78 & 0.75 & 0.05 & -0.8767 \\
\hline 47 & AUDJPY & $3 Y$ & -0.54 & 0.93 & 0.50 & 0.07 & -0.9191 \\
\hline 48 & AUDJPY & $4 Y$ & -0.55 & 1.26 & 0.38 & 0.09 & -0.9569 \\
\hline 49 & AUDJPY & $5 \mathrm{Y}$ & -0.58 & 2.44 & 0.30 & 0.18 & -0.9819 \\
\hline 50 & USDTRY & $3 \mathrm{M}$ & 0.60 & 0.89 & 6.02 & 0.04 & -0.3920 \\
\hline 51 & USDTRY & $6 \mathrm{M}$ & 0.63 & 0.61 & 3.02 & 0.04 & -0.3507 \\
\hline 52 & USDTRY & $1 Y$ & 0.70 & 0.39 & 1.50 & 0.04 & -0.2110 \\
\hline 53 & USDTRY & $2 \mathrm{Y}$ & 0.76 & 0.23 & 0.75 & 0.04 & 0.1342 \\
\hline 54 & USDTRY & $3 \mathrm{Y}$ & 0.78 & 0.17 & 0.50 & 0.05 & 0.7301 \\
\hline 55 & USDTRY & $4 Y$ & 0.77 & 0.14 & 0.38 & 0.05 & 0.9388 \\
\hline 56 & USDTRY & $5 \mathrm{Y}$ & 0.78 & 0.15 & 0.30 & 0.06 & 0.6000 \\
\hline
\end{tabular}

Table 2. Interest rates

\begin{tabular}{ccc}
\hline currency & symbol & interest rate \\
\hline Australian Dollar & AUD & 0.0650 \\
US Dollar & USD & 0.0200 \\
Euro & EUR & 0.0425 \\
British Pound & GBP & 0.0500 \\
Japanese Yen & JPY & 0.0050 \\
Brazilian Real & BRL & 0.1375 \\
Turkish Lira & TRY & 0.1675 \\
\hline
\end{tabular}


approximation of $U(T)$ in the the HV scheme, we use the theoretical result $\theta=\frac{1}{2}+\frac{\sqrt{3}}{6}$ in [22, Conjecture 2.9] and a time step $\Delta t=T / 1000$ in (19).

For efficiency one could use much less points in the $v$-direction than in the $s$ direction. In our experiments, we take $m_{1}=2 m_{2}$. For each case in Table 1 , the global spatial errors $e\left(2 m_{2}, m_{2}\right)$ are plotted against $1 / m_{2}$ for $m_{2}=10,20, \ldots, 100$. The numerical order of convergence $p$ of the spatial discretization is determined by a linear least square fit of the outcomes for the global spatial errors. For each case of the butterfly and strangle options given by the parameters in the Table 1, we present the calculated values of $p$ in Table 3 for $m_{2}=100$ and suitably chosen $S_{\max }$ and $V_{\max }$. The column spatial error represents maximum absolute error $e\left(m_{1}, m_{2}\right)$.

The FD discretization described in Section 4.1 performs satisfactorily well and numerical order of convergence $p \approx 2$ is obtained in almost all cases of the parameter sets from Table 1. Slightly lower order of convergence is found in some cases, namely, cases 10-14 and 44-47 for the butterfly and case 49 for the strangle, which may be attributed to the strong violation of the Feller condition. These cases do not give problems for the butterfly and strangle at the same time owing to their dissimilar payoff functions and mismatched boundary values. As a final remark, we observe that a sufficiently large choice of $S_{\max }$ and $V_{\max }$ is necessary for a regular (monotone) convergence behaviour of the spatial discretization error as a function of $m_{1}=2 m_{2}$.

\subsection{Time-discretization error}

Numerical experiments to examine the important stability and convergence behaviours of the MCS scheme in applications to semi-discrete Heston PDEs (16), where the Feller condition is (strongly) violated, is performed in this section. In addition to the MCS scheme, the HV scheme with $\theta=\frac{1}{2}+\frac{\sqrt{3}}{6}$ is also used in the experiments for comparison.

First recall the $\gamma$-condition on the diffusion coefficients $\left(d_{i j}\right)$ for unconditional stability of the MCS scheme applied to $2 \mathrm{D}$ convection-diffusion problems with mixed derivatives in the von Neumann frame-work [26]

$$
\left|d_{i j}\right| \leq \gamma \sqrt{d_{i i} d_{j j}} \quad i, j=1,2 i \neq j .
$$

Under this condition, the bounds of the parameter $\theta$, such that the MCS scheme is unconditionally stable can be computed by the following theorem in [26] for any given $\gamma \in[0,1]:$

Theorem 5.1. Denote

$$
\begin{aligned}
& f_{1}(\theta):=\theta \sqrt{\frac{5-2 \theta}{1-2 \theta}}-\theta, \\
& g_{1}(\theta):=\frac{\theta}{2}\left(\frac{2 \theta+\sqrt{4 \theta^{2}-16 \theta+6}}{1-2 \theta}\right), \\
& f_{2}(\theta):=\frac{4 \theta^{2}}{\sqrt{4 \theta^{4}-24 \theta^{3}+24 \theta^{2}-8 \theta+1}-2 \theta^{2}+4 \theta-1} \quad \text { and } \\
& g_{2}(\theta):=\frac{-4 \theta^{3}+11 \theta^{2}-6 \theta+1+\sqrt{16 \theta^{6}-128 \theta^{5}+216 \theta^{4}-160 \theta^{3}+61 \theta^{2}-12 \theta+1}}{5 \theta^{2}-4 \theta+1} .
\end{aligned}
$$

When applied to a two-dimensional evolutionary convection-diffusion equation with 
Table 3. Spatial discretization

\begin{tabular}{|c|c|c|c|c|c|c|c|c|}
\hline \multirow[b]{2}{*}{ Case No. } & \multicolumn{4}{|l|}{ butterfly } & \multicolumn{4}{|l|}{ strangle } \\
\hline & Order $p$ & Spatial error & $S_{\max }$ & $V_{\max }$ & Order $p$ & Spatial error & $S_{\max }$ & $V_{\max }$ \\
\hline 01 & 1.9 & $5.0 \times 10^{-3}$ & $8 \mathrm{~K}$ & 5 & 1.9 & $4.0 \times 10^{-3}$ & $10 \mathrm{~K}$ & 5 \\
\hline 02 & 1.9 & $4.7 \times 10^{-3}$ & $10 \mathrm{~K}$ & 5 & 1.9 & $6.6 \times 10^{-3}$ & $10 \mathrm{~K}$ & 5 \\
\hline 03 & 1.9 & $4.4 \times 10^{-3}$ & $10 \mathrm{~K}$ & 5 & 1.9 & $1.0 \times 10^{-2}$ & $10 \mathrm{~K}$ & 5 \\
\hline 04 & 1.9 & $3.8 \times 10^{-3}$ & $10 \mathrm{~K}$ & 5 & 2.0 & $1.3 \times 10^{-2}$ & $10 \mathrm{~K}$ & 5 \\
\hline 05 & 1.9 & $3.6 \times 10^{-3}$ & $10 \mathrm{~K}$ & 5 & 2.1 & $1.2 \times 10^{-2}$ & $10 \mathrm{~K}$ & 5 \\
\hline 06 & 2.0 & $3.4 \times 10^{-3}$ & $10 \mathrm{~K}$ & 5 & 2.1 & $1.5 \times 10^{-2}$ & $15 \mathrm{~K}$ & 5 \\
\hline 07 & 1.9 & $4.0 \times 10^{-3}$ & $10 \mathrm{~K}$ & 5 & 2.1 & $1.5 \times 10^{-2}$ & $15 \mathrm{~K}$ & 5 \\
\hline 08 & 1.8 & $5.9 \times 10^{-3}$ & $12 \mathrm{~K}$ & 5 & 2.1 & $3.0 \times 10^{-3}$ & $10 \mathrm{~K}$ & 5 \\
\hline 09 & 1.8 & $8.3 \times 10^{-3}$ & $15 \mathrm{~K}$ & 5 & 2.0 & $6.0 \times 10^{-3}$ & $10 \mathrm{~K}$ & 5 \\
\hline 10 & 1.7 & $1.2 \times 10^{-2}$ & $25 \mathrm{~K}$ & 15 & 2.0 & $7.9 \times 10^{-3}$ & $10 \mathrm{~K}$ & 5 \\
\hline 11 & 1.6 & $1.5 \times 10^{-2}$ & $25 \mathrm{~K}$ & 15 & 2.0 & $1.0 \times 10^{-2}$ & $10 \mathrm{~K}$ & 5 \\
\hline 12 & 1.6 & $1.5 \times 10^{-2}$ & $25 \mathrm{~K}$ & 15 & 2.0 & $1.1 \times 10^{-2}$ & $10 \mathrm{~K}$ & 5 \\
\hline 13 & 1.7 & $1.4 \times 10^{-2}$ & $25 \mathrm{~K}$ & 15 & 2.0 & $1.3 \times 10^{-2}$ & $10 \mathrm{~K}$ & 5 \\
\hline 14 & 1.7 & $1.3 \times 10^{-2}$ & $25 \mathrm{~K}$ & 15 & 1.9 & $1.4 \times 10^{-2}$ & $10 \mathrm{~K}$ & 5 \\
\hline 15 & 1.9 & $5.1 \times 10^{-3}$ & $8 \mathrm{~K}$ & 5 & 1.9 & $3.9 \times 10^{-3}$ & $10 \mathrm{~K}$ & 5 \\
\hline 16 & 1.9 & $4.6 \times 10^{-3}$ & $10 \mathrm{~K}$ & 5 & 1.9 & $6.6 \times 10^{-3}$ & $10 \mathrm{~K}$ & 5 \\
\hline 17 & 1.9 & $4.3 \times 10^{-3}$ & $10 \mathrm{~K}$ & 5 & 1.9 & $1.0 \times 10^{-2}$ & $10 \mathrm{~K}$ & 5 \\
\hline 18 & 2.0 & $4.0 \times 10^{-3}$ & $10 \mathrm{~K}$ & 5 & 2.0 & $1.3 \times 10^{-2}$ & $10 \mathrm{~K}$ & 5 \\
\hline 19 & 2.0 & $3.8 \times 10^{-3}$ & $10 \mathrm{~K}$ & 5 & 2.1 & $1.3 \times 10^{-2}$ & $10 \mathrm{~K}$ & 5 \\
\hline 20 & 2.0 & $3.6 \times 10^{-3}$ & $10 \mathrm{~K}$ & 5 & 2.1 & $1.5 \times 10^{-2}$ & $15 \mathrm{~K}$ & 5 \\
\hline 21 & 2.0 & $3.9 \times 10^{-3}$ & $10 \mathrm{~K}$ & 5 & 2.1 & $1.5 \times 10^{-2}$ & $15 \mathrm{~K}$ & 5 \\
\hline 22 & 1.9 & $5.4 \times 10^{-2}$ & $50 \mathrm{~K}$ & 25 & 2.0 & $4.1 \times 10^{-3}$ & $10 \mathrm{~K}$ & 5 \\
\hline 23 & 1.9 & $5.4 \times 10^{-3}$ & $10 \mathrm{~K}$ & 5 & 1.9 & $7.1 \times 10^{-3}$ & $10 \mathrm{~K}$ & 5 \\
\hline 24 & 1.9 & $5.4 \times 10^{-3}$ & $10 \mathrm{~K}$ & 5 & 1.9 & $1.0 \times 10^{-2}$ & $10 \mathrm{~K}$ & 5 \\
\hline 25 & 1.9 & $5.6 \times 10^{-3}$ & $10 \mathrm{~K}$ & 5 & 2.0 & $1.4 \times 10^{-2}$ & $10 \mathrm{~K}$ & 5 \\
\hline 26 & 1.9 & $5.2 \times 10^{-3}$ & $10 \mathrm{~K}$ & 5 & 2.1 & $1.2 \times 10^{-2}$ & $10 \mathrm{~K}$ & 5 \\
\hline 27 & 1.9 & $5.0 \times 10^{-3}$ & $10 \mathrm{~K}$ & 5 & 2.1 & $1.5 \times 10^{-2}$ & $15 \mathrm{~K}$ & 5 \\
\hline 28 & 1.9 & $4.7 \times 10^{-3}$ & $10 \mathrm{~K}$ & 5 & 2.1 & $1.4 \times 10^{-2}$ & $18 \mathrm{~K}$ & 5 \\
\hline 29 & 1.9 & $4.8 \times 10^{-3}$ & $25 \mathrm{~K}$ & 15 & 2.0 & $3.5 \times 10^{-3}$ & $10 \mathrm{~K}$ & 5 \\
\hline 30 & 1.9 & $5.2 \times 10^{-3}$ & $25 \mathrm{~K}$ & 15 & 1.9 & $5.9 \times 10^{-3}$ & $10 \mathrm{~K}$ & 5 \\
\hline 31 & 1.9 & $5.8 \times 10^{-3}$ & $25 \mathrm{~K}$ & 15 & 2.0 & $8.5 \times 10^{-3}$ & $10 \mathrm{~K}$ & 5 \\
\hline 32 & 1.9 & $5.8 \times 10^{-3}$ & $25 \mathrm{~K}$ & 15 & 2.0 & $1.2 \times 10^{-2}$ & $10 \mathrm{~K}$ & 5 \\
\hline 33 & 2.1 & $5.2 \times 10^{-3}$ & $25 \mathrm{~K}$ & 15 & 2.1 & $1.2 \times 10^{-2}$ & $10 \mathrm{~K}$ & 5 \\
\hline 34 & 2.2 & $4.9 \times 10^{-3}$ & $25 \mathrm{~K}$ & 15 & 2.1 & $1.5 \times 10^{-2}$ & $15 \mathrm{~K}$ & 5 \\
\hline 35 & 2.2 & $4.5 \times 10^{-3}$ & $25 \mathrm{~K}$ & 15 & 2.1 & $1.4 \times 10^{-2}$ & $15 \mathrm{~K}$ & 5 \\
\hline 36 & 1.9 & $6.2 \times 10^{-3}$ & $8 \mathrm{~K}$ & 5 & 2.0 & $4.1 \times 10^{-3}$ & $10 \mathrm{~K}$ & 5 \\
\hline 37 & 1.9 & $7.8 \times 10^{-3}$ & $10 \mathrm{~K}$ & 5 & 1.9 & $6.9 \times 10^{-3}$ & $10 \mathrm{~K}$ & 5 \\
\hline 38 & 2.0 & $8.1 \times 10^{-3}$ & $10 \mathrm{~K}$ & 5 & 1.9 & $1.0 \times 10^{-2}$ & $10 \mathrm{~K}$ & 5 \\
\hline 39 & 2.0 & $7.9 \times 10^{-3}$ & $10 \mathrm{~K}$ & 5 & 2.0 & $1.0 \times 10^{-2}$ & $10 \mathrm{~K}$ & 5 \\
\hline 40 & 2.0 & $7.2 \times 10^{-3}$ & $10 \mathrm{~K}$ & 5 & 1.9 & $1.4 \times 10^{-2}$ & $15 \mathrm{~K}$ & 5 \\
\hline 41 & 1.9 & $1.0 \times 10^{-2}$ & $12 \mathrm{~K}$ & 5 & 1.9 & $2.0 \times 10^{-2}$ & $20 \mathrm{~K}$ & 15 \\
\hline 42 & 1.9 & $1.0 \times 10^{-2}$ & $12 \mathrm{~K}$ & 5 & 1.7 & $1.8 \times 10^{-2}$ & $25 \mathrm{~K}$ & 15 \\
\hline 43 & 1.9 & $5.8 \times 10^{-3}$ & $25 \mathrm{~K}$ & 15 & 1.9 & $3.5 \times 10^{-3}$ & $10 \mathrm{~K}$ & 5 \\
\hline 44 & 1.7 & $7.4 \times 10^{-3}$ & $25 \mathrm{~K}$ & 15 & 1.9 & $5.6 \times 10^{-3}$ & $10 \mathrm{~K}$ & 5 \\
\hline 45 & 1.6 & $8.5 \times 10^{-3}$ & $25 \mathrm{~K}$ & 15 & 1.9 & $9.1 \times 10^{-2}$ & $10 \mathrm{~K}$ & 5 \\
\hline 46 & 1.7 & $6.2 \times 10^{-3}$ & $25 \mathrm{~K}$ & 15 & 2.0 & $1.1 \times 10^{-2}$ & $10 \mathrm{~K}$ & 5 \\
\hline 47 & 1.7 & $6.0 \times 10^{-3}$ & $25 \mathrm{~K}$ & 15 & 2.0 & $1.2 \times 10^{-2}$ & $18 \mathrm{~K}$ & 10 \\
\hline 48 & 1.9 & $5.5 \times 10^{-3}$ & $25 \mathrm{~K}$ & 15 & 2.0 & $9.1 \times 10^{-3}$ & $18 \mathrm{~K}$ & 10 \\
\hline 49 & 1.9 & $6.5 \times 10^{-3}$ & $25 \mathrm{~K}$ & 15 & 1.6 & $1.7 \times 10^{-2}$ & $50 \mathrm{~K}$ & 25 \\
\hline 50 & 1.9 & $7.6 \times 10^{-3}$ & $8 \mathrm{~K}$ & 5 & 2.0 & $3.6 \times 10^{-3}$ & $10 \mathrm{~K}$ & 5 \\
\hline 51 & 1.9 & $9.3 \times 10^{-3}$ & $8 \mathrm{~K}$ & 5 & 1.9 & $7.1 \times 10^{-3}$ & $10 \mathrm{~K}$ & 5 \\
\hline 52 & 1.9 & $1.2 \times 10^{-2}$ & $8 \mathrm{~K}$ & 5 & 1.9 & $1.0 \times 10^{-2}$ & $10 \mathrm{~K}$ & 5 \\
\hline 53 & 1.9 & $1.4 \times 10^{-2}$ & $10 \mathrm{~K}$ & 5 & 2.0 & $1.0 \times 10^{-2}$ & $10 \mathrm{~K}$ & 5 \\
\hline 54 & 2.0 & $1.3 \times 10^{-2}$ & $10 \mathrm{~K}$ & 5 & 2.0 & $1.1 \times 10^{-2}$ & $15 \mathrm{~K}$ & 5 \\
\hline 55 & 2.1 & $1.1 \times 10^{-2}$ & $10 \mathrm{~K}$ & 5 & 2.1 & $9.3 \times 10^{-3}$ & $18 \mathrm{~K}$ & 5 \\
\hline 56 & 2.1 & $1.1 \times 10^{-2}$ & $10 \mathrm{~K}$ & 5 & 2.1 & $8.9 \times 10^{-3}$ & $22 \mathrm{~K}$ & 5 \\
\hline
\end{tabular}


mixed derivative and constant coefficients, the MCS scheme is unconditionally stable, in the von Neumann sense, whenever

$$
\gamma \leq \min \left\{f_{1}(\theta), g_{1}(\theta)\right\}, \quad \text { for } \quad \frac{1}{4} \leq \theta \leq \frac{1}{3}
$$

and

$$
\gamma \leq \min \left\{f_{2}(\theta), g_{2}(\theta)\right\}, \quad \text { for } \quad \frac{1}{3} \leq \theta \leq \frac{1}{2}
$$

For a rigorous proof, see [26]. Since the diffusion matrix for the Heston PDE is $\left(\begin{array}{cc}\frac{1}{2} s^{2} v & \frac{1}{2} \rho \sigma s v \\ \frac{1}{2} \rho \sigma s v & \frac{1}{2} \sigma^{2} v\end{array}\right)$ one can take $\gamma$ equal to $|\rho|$.

A suitable damping procedure is employed to avoid relatively larger temporal errors for modest time step $\Delta t$, cf. [28]. First apply, at $t=0$, two backward Euler steps with step size $\Delta t / 2$ and subsequently proceed with the MCS (correspondingly HV) scheme from $t=\Delta t$ onwards.

Analogous to the global spatial discretization error, define global time-discretization error at time $t=T=N \Delta t$ by

$$
\hat{e}\left(N ; m_{1}, m_{2}\right)=\max \left\{\left|U_{k}(T)-U_{N, k}\right|: \frac{K}{2}<s_{i}<\frac{3 K}{2}, 0<v_{j}<1\right\} .
$$

Here the components $U_{k}(T)$ and $U_{N, k}$ correspond to the spatial grid point $\left(s_{i}, v_{j}\right)$. The $(s, v)$-domain of the global temporal error is same as that of the global spatial error. The error is measured in the maximum norm. Global temporal errors $\hat{e}\left(N ; m_{1}, m_{2}\right)$ for $m_{1}=2 m_{2}=100$ is computed for a range of numbers $N$ between 1 and 1000 . A reference value for $U(T)$ is obtained by application of the HV scheme with $N=5000$.

We investigated how the choice of $\theta$ affects the convergence behavior of the MCS scheme, and compared the MCS results with that of the HV scheme. The HV scheme is implemented for a single value of $\theta=\frac{1}{2}+\frac{\sqrt{3}}{6}$, whereas the $\theta$ for the MCS scheme take three different values, starting with $\theta=\frac{1}{4}$, the minimum prescribed in Theorem 5.1, $\theta=\frac{1}{3}$, a commonly used value in the literature (cf. [18]), and then $\theta=\frac{2}{3}$. Figures 3 and 4 display the plots of $\hat{e}$ vs. $1 / N$, corresponding to $m_{1}=2 m_{2}=100$, for representative cases selected from the 56 cases in Table 1 . It is evident that an increase in $\theta$ causes either a growth of the error constant $C$, or has negligible effect. It is observed that in the special cases where the Feller condition is strongly violated (13, 14, 48 and 49), the MCS scheme outperforms the HV scheme. In all other cases, the MCS scheme performs as well as the HV scheme, and both show a convergence of order 2 .

The experiments are repeated to compute $\hat{e}\left(N ; m_{1}, m_{2}\right)$ for $m_{1}=2 m_{2}=200$. The obtained results are almost the same as those for $m_{1}=2 m_{2}=100$ for all permissible $\theta$. For both MCS and HV schemes, the temporal discretization errors are bounded from above by a moderate value and decay monotonically as $\Delta t$ decreases, and it is true irrespective of the choice of $m_{1}, m_{2}$.

It is worth noting that the convergence behavior of the MCS scheme is of the form $C(\Delta t)^{2}(0<\Delta t \leq \tau)$, where the constants $C, \tau>0$ are only weakly dependent on the number of spatial grid points. In other words, the scheme shows a general order of convergence equal to 2 .

Instabilities due to smaller $\theta$ is presented for a few representative test cases in Figure 5 . It can be seen that the necessary lower bounds on $\theta$ are sharp for the MCS scheme 


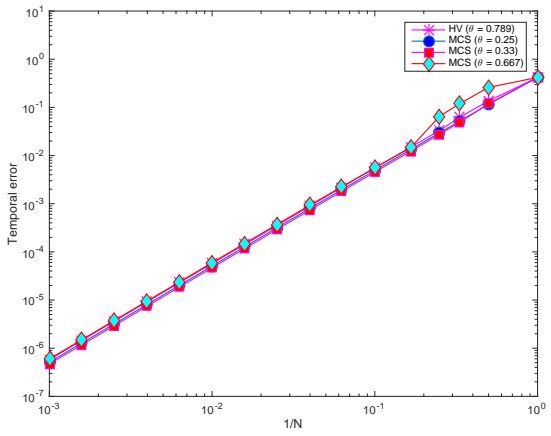

(a) Case 1

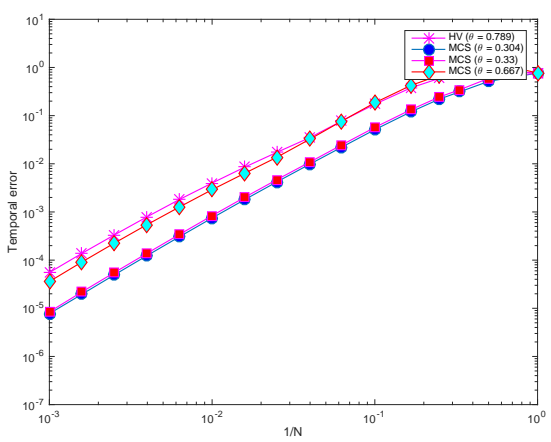

(c) Case 13

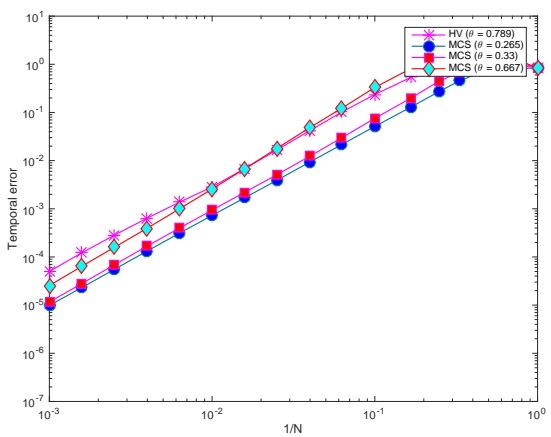

(e) Case 48

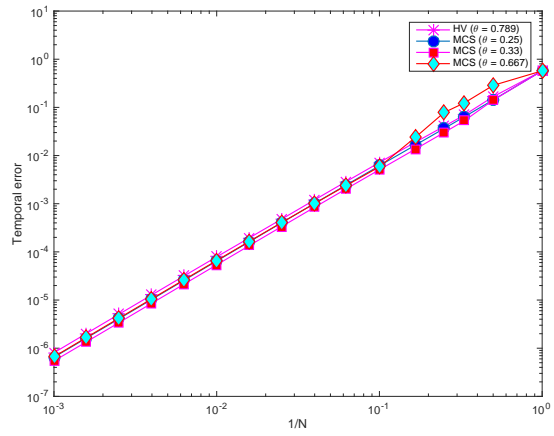

(b) Case 2

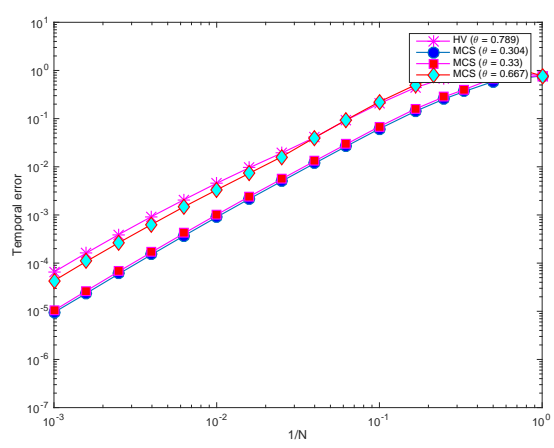

(d) Case 14

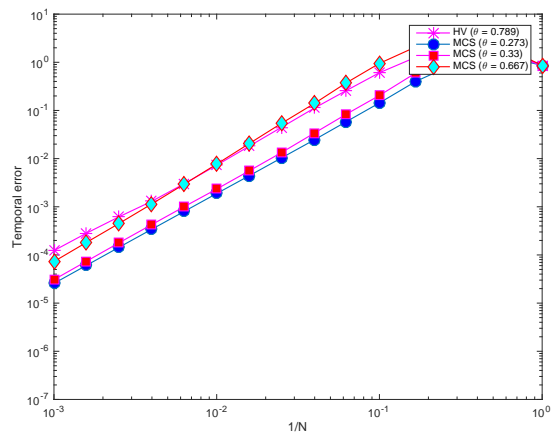

(f) Case 49

Figure 3. Global temporal errors $\hat{e}(N ; 100,50)$ versus $1 / N$ for MCS and HV schemes for butterfly options.

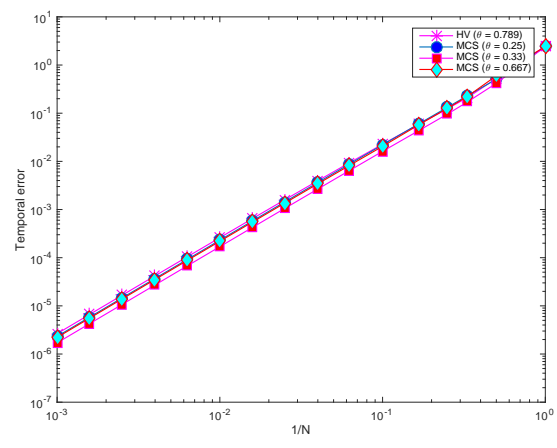

(a) Case 1

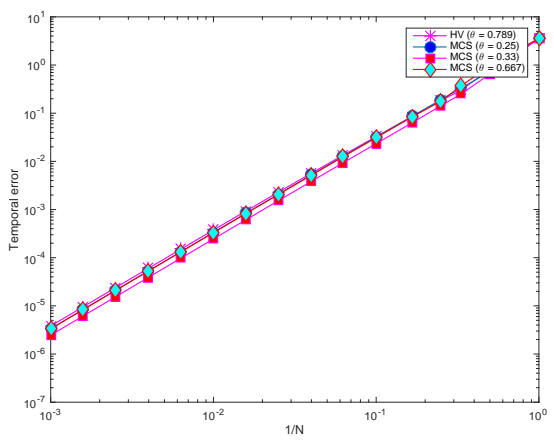

(b) Case 2 


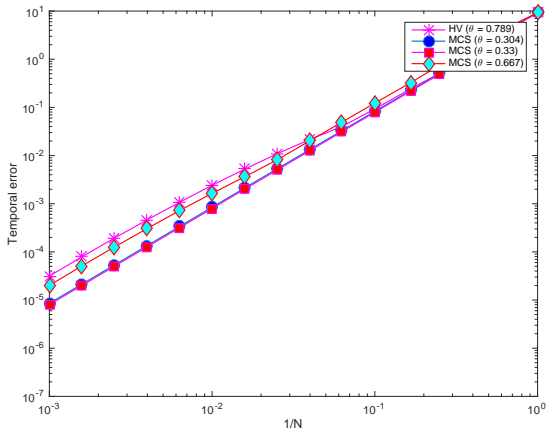

(c) Case 13

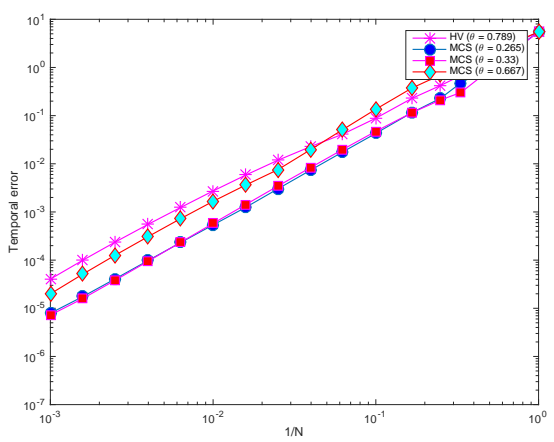

(e) Case 48

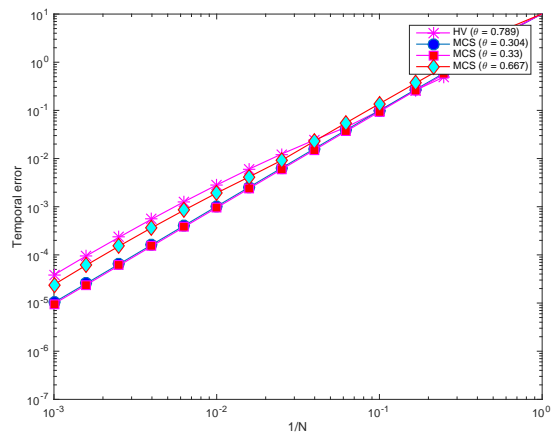

(d) Case 14

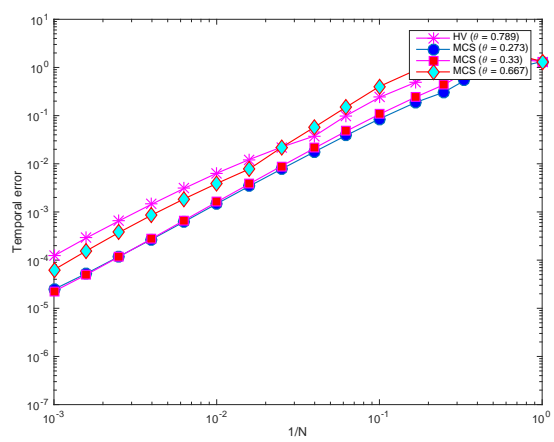

(f) Case 49

Figure 4. Global temporal errors $\hat{e}(N ; 100,50)$ versus $1 / N$ for MCS and HV schemes for strangle options.

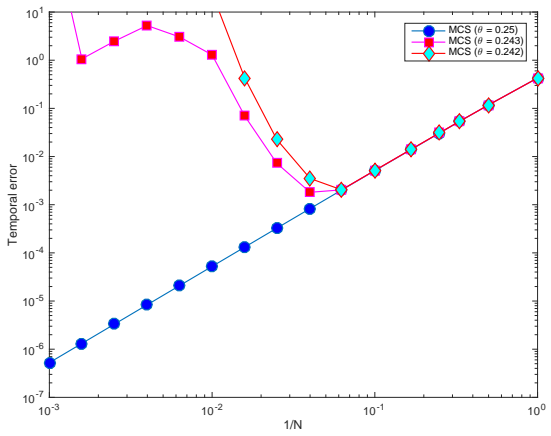

(a) Case $1, m=200$

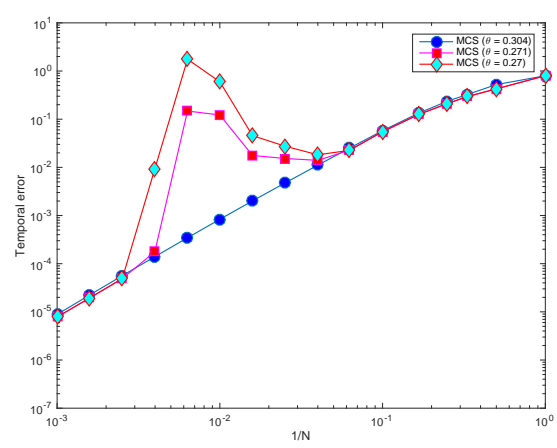

(c) Case $13, m=200$

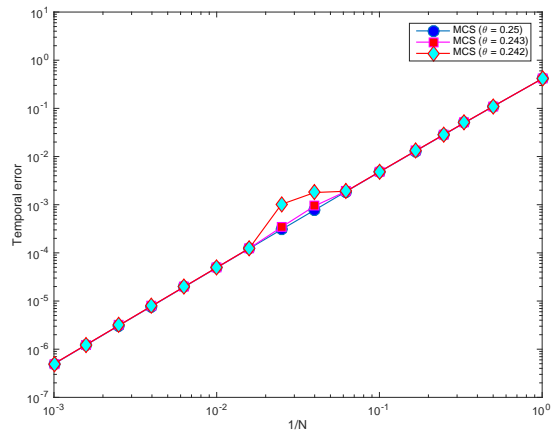

(b) Case 1, $m=100$

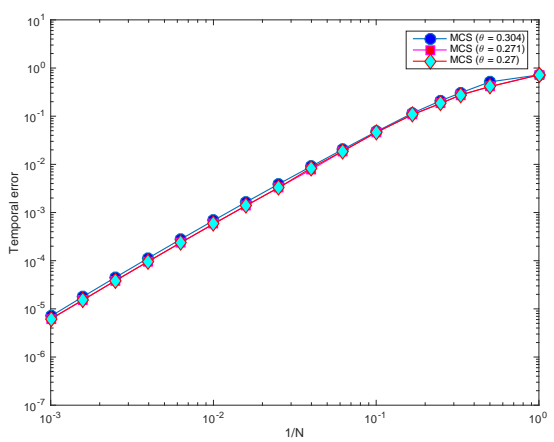

(d) Case 13, $m=100$ 


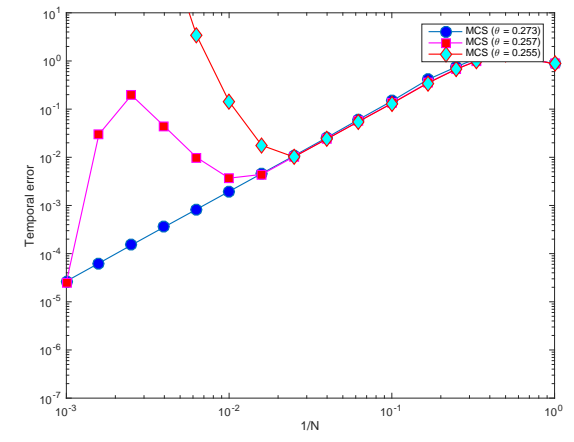

(e) Case $49, m=200$

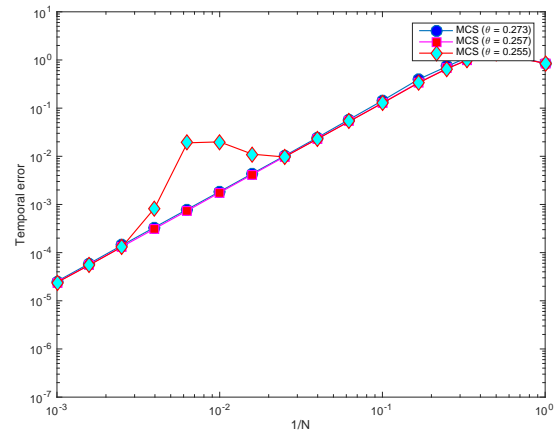

(f) Case 49, $m=100$

Figure 5. Global temporal errors $\hat{e}(N ; m, m / 2)$ versus $1 / N$ for minimum prescribed values of $\theta$ for the MCS scheme for butterfly options, compared with slightly smaller $\theta$.

in confirmation with the von Neumann stability results. Even a slightly smaller choice of $\theta$ ( 2 to $10 \%$ less than the minimum prescribed, corresponding to a given $\gamma$ ) would yield instability for almost all cases. On a finer spatial grid $\left(m_{1}=2 m_{2}=200\right)$ the instability becomes clearly more visible as shown in the figure. The global temporal error increases rapidly with a marginal decrease in $\theta$. There is a similar trend for the strangle options, but due to the space limitation we omit the graphs.

\section{Conclusions}

In this paper, we examined the application of the modified Craig-Sneyd scheme in pricing foreign exchange options through case studies of 56 options on 8 currency pairs. Although all the required conditions of von Neumann analysis have been violated the MCS scheme exhibits an unconditionally stable behaviour in our numerical experiments. The theoretical stability bounds for the MCS scheme seem to be quite sharp, even though a slight violation of the minimum prescribed value could lead to undesired solutions. The convergence rates of both the spatial discretization errors and the temporal discretization errors are satisfactory for the non-smooth initial conditions. Extra care should be taken, however, when dealing with cases where the Feller condition is strongly violated. In these special cases increasing the spatial domain is often effective, and the MCS scheme seems to be more accurate compared with the HV scheme. Our case study proves that the MCS ADI scheme is robust and reliable for pricing FX options under the Heston model with real market data.

\section{References}

[1] L. B. Andersen \& V. V. Pieterbarg, Interest rate modeling, Volume I: Foundations and Vanilla Models. First edn. Atlantic Financial Press (2010).

[2] Z. Bučková, M. Ehrhardt \& M. Günther, Fichera Theory and its Application in Finance. Proceedings of the 18th European Conference on Mathematics for Industry, June 9-13, 2014, Taormina, Italy, Springer Verlag, Berlin, Heidelberg, (2015).

[3] I. J. Clark, Foreign exchange option pricing -A practitioner's guide. Wiley UK (2011).

[4] I. J. Craig \& A. D. Sneyd, An alternating-direction implicit scheme for parabolic equations with mixed derivatives, Compt. Math. Appl. 16 (1988) 341-350. 
[5] D-M. Dang, C. Christara, K. R. Jackson \& A. Lakhani, An efficient numerical PDE approach for pricing foreign exchange interest rate hybrid derivatives, J. Comput. Financ. 18 (2015) 39-93.

[6] J. Douglas Jr., Alternating direction methods for three space variables, Numer. Math 4 (1962) 41-63.

[7] D. Duffy, Finite difference methods in financial enginerring: a aprtial differential equation approach. Wiley Finance (2006).

[8] W. Feller, Two Singular Diffusion Problems, Ann. of Math, 54 (1) 173-182 (1951).

[9] G. Fichera, On a unified theory of boundary value problems for elliptic-parabolic equations of second order in boundary value problems, ed. R.E. Langer, Univ. of Wisc. Press (1960).

[10] T. Haentjens \& K. J. in 't Hout, Alternating direction implicit finite difference schemes for the Heston-Hull-White partial differential equation, J. Comp. Finan., 16 (2012) 83110 .

[11] T. Haentjens \& K. J. in 't Hout, ADI schemes for pricing American options under the Heston model., Appl. Math. Finan. 22, 207-237 (2015).

[12] C. Hendricks, C. Heuer, M. Ehrhardt \& M. Günther, High-order ADI finite difference schemes for parabolic equations in the combination technique with application in finance, J. Comput. Appl. Math., 316 (2017) 175-194.

[13] C. Hendricks, Sparse Grid Techniques for Pricing Energy Derivatives, Doctoral Thesis at the Bergische Universität Wuppertal (2017).

[14] S. L. Heston, A closed-form solution for options with stochastic volatility with applications to bond and currency options, Rev. Fin. Stud. 6(2) (1993) 327-343.

[15] W. Hundsdorfer, Accuracy and stability of splitting with Stabilizing Corrections, Appl. Numer. Math., 42 (2002) 213-233.

[16] W. Hundsdorfer \& J. G. Verwer, Numerical Solution of Time-Dependent AdvectionDiffusion-Reaction Equations. Springer-Verlag, Berlin (2003).

[17] S. Ikonen \& J. Toivanen, Componentwise splitting methods for pricing American options under stochastic volatility, Int. J. Theor. Appl. Finan. 10 (2007) 331-361.

[18] K. J. in 't Hout \& S. Foulon, ADI finite difference schemes for option pricing in the Heston model with correlation, Int. J. Num. Anal. Mod. 7 (2010) 303-320.

[19] K. J. in 't Hout \& C. Mishra, Stability of the modified Craig-Sneyd scheme for twodimensional convection-diffusion equations with mixed derivative term, Math. Comp. Simul. 81 (2011) 2540-2548.

[20] K. J. in 't Hout \& C. Mishra, Stability of ADI schemes for multidimensional diffusion equations with mixed derivative terms, Appl. Numer. Math. 74 (2013) 83-94.

[21] K. J. in 't Hout \& J. Toivanen, ADI schemes for valuing European options under the Bates model, Appl. Numer. Math. 130, (2018) 143-156.

[22] K. J. in 't Hout \& B. D. Welfert, Stability of ADI schemes applied to convection-diffusion equations with mixed derivative terms, Appl. Num. Math. 57 (2007) 19-35.

[23] K. J. in 't Hout \& B. D. Welfert, Unconditional stability of second-order ADI schemes applied to multi-dimensional diffusion equations with mixed derivative terms, Appl. Num. Math. 59 (2009) 677-692.

[24] A. Itkin \& P. Carr, Jumps without tears: A new splitting technology for barrier options., Int. J. Numer. Anal. Mod., 8 (2011) 667-704.

[25] A. Lipton, Mathematical Methods for Foreign Exchange: A Financial Engineer's Approach. World Scientific, Singapore (2001).

[26] C. Mishra, A new stability result for the modified Craig-Sneyd scheme applied to twodimensional convection-diffusion equations with mixed derivatives, Appl. Math. Comput., 285 (2016) 41-50.

[27] K. W. Morton \& David Mayers, Numerical Solution of Partial Differential Equations, 2nd Ed., Cambridge University Press (2005).

[28] R. Rannacher, Finite element solution of diffusion problems with irregular data, Numer. Math. 43 (1984) 309-327.

[29] D. Tavella \& C. Randall, Pricing financial instruments. Wiley, New York (2000). 
[30] G. Winkler, T. Apel \& U. Wystup, Valuation of options in Heston stochastic volatility model using finite element method, in: Foreign Exchange Risk, eds. J. Hakala \& U. Wystup. Risk Publ., 2002.

[31] S-P. Zhu \& W-T. Chen, A predictor-corrector scheme based on the ADI method for pricing American puts with stochastic volatility, Comput. Math. Appl., 62 (2011) 1-26. 